\title{
Use of Diagnosis Code in Mental Health Syndrome Definition
}

\author{
Achintya N. Dey*, Deborah Gould, Nelson Adekoya, Peter Hicks, Girum S. Ejigu, \\ Roseanne English, Jenny Couse and Hong Zhou
}

CCSELS/DHIS, CDC, Atlanta, GA, USA

\section{Objective}

The objectives of this study are to (1) create a mental health syndrome definition for syndromic surveillance to monitor mental health-related ED visits in near real time; (2) examine whether CC data alone can accurately detect mental health related ED visits; and (3) assess the added value of using Dx data to detect mental healthrelated ED visits.

\section{Introduction}

Between 2006 and 2013, the rate of emergency department (ED) visits related to mental and substance use disorders increased substantially. This increase was higher for mental disorders visits (55 percent for depression, anxiety or stress reactions and 52 percent for psychoses or bipolar disorders) than for substance use disorders (37 percent) visits [1]. This increasing number of ED visits by patients with mental disorders indicates a growing burden on the health-care delivery system. New methods of surveillance are needed to identify and understand these changing trends in ED utilization and affected underlying populations.

Syndromic surveillance can be leveraged to monitor mental health-related ED visits in near real-time. ED syndromic surveillance systems primarily rely on patient chief complaints (CC) to monitor and detect health events. Some studies suggest that the use of ED discharge diagnoses data (Dx), in addition to or instead of CC, may improve sensitivity and specificity of case identification [2].

\section{Methods}

We extracted a de-identified random sample of 50,000 ED visits with CC from the National Syndromic Surveillance Program (NSSP) for the period January 1-June 30, 2017. NSSP's BioSense Platform receives ED data from $>4000$ hospitals, representing about 55 percent of all ED visits in the country [3]. From this sample we extracted 22868 ED visits that included both CC and Dx data. We then applied our mental health syndrome case definition which comprised mental health-related keywords and ICD-9-CM and ICD$10-C M$ codes. We queried CC text for the words "stress," "PTSD," "anxiety," "depression," "clinical depression," "manic depression," "unipolar depression," "agitated," "nervousness," "mental health," "mental disorder," "affective disorder," "schizoaffective disorder," "psycoaffective disorder," "obsessive-compulsive disorder," "mood disorder," "bipolar disorder," "schizotypal personality disorder," "panic disorder," "psychosis," "paranoia," "psych," "manic," "mania," "hallucinating," "hallucination," "mental episode," and "mental illness." We queried Dx fields either for ICD-9- CM codes 295-296; 300, 311 or for ICD-10-CM codes F20-F48. The ICD-9$\mathrm{CM}$ and ICD-10-CM codes used to identify mental health-related ED visits are based on the mental health disorders most frequently seen in EDs. Alcohol and substance use, suicide ideation, and suicide attempt were excluded from this study because they are included in alternate syndromes [2]. We manually reviewed the $\mathrm{CC}$ text to validate the search terms. Sensitivity, specificity, and positive predictive value will be calculated based on agreement of coding mental health against the human review of mental health visits.
Based on our case definition, the sample of 22868 ED visits with $\mathrm{CC}$ and Dx data was further stratified into two groups: (1) mental health identified in either CC or Dx, and (2) no mental health identified in CC and Dx. Group 1 was further stratified into three groups: (a) mental health identified only in CC, (b) mental health identified in both CC and Dx, and (c) mental health identified only in Dx. The sample of 27132 ED visits with CC and no Dx data was further stratified into two groups: (1) mental health identified in $\mathrm{CC}$, and (2) no mental health identified in CC (Figure).

\section{Results}

Of the 50,000 sample of ED visits with CC data, 22868 visits had both CC and Dx data. Of the 22868 visits, we identified 1560 mental health-related ED visits using the mental health syndrome case definition. Of those visits, 241 were identified by a CC only, 226 were identified by both CC and Dx, and 1093 by a mental health-related Dx. Of the 27132 ED visits without Dx data, 421 had mental health identified in $\mathrm{CC}$.

\section{Conclusions}

Based on our preliminary analysis these findings suggest potential benefits of including Dx data in syndrome binning for mental health. Mental health terms are more likely to be found in Dx data than in the CC (1093 vs. 662). Using CC alone may underestimate the number of mental health-related ED visits. This study had several limitations. Not all facilities reporting to NSSP provide chief complaint data in the same manner, some provide $\mathrm{CC}$ as a drop down menu with predefined terms while others include the full text of CC. Not all records contained a Dx code which limited our ability to examine the added value of Dx code for that subset.

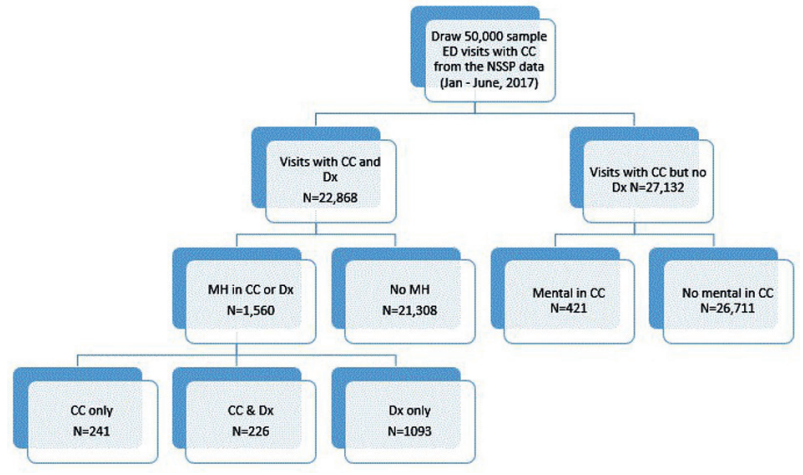

Keywords

Emergency department; Mental health; Syndromic surveillance 


\section{ISDS 2018 Conference Abstracts}

\section{References}

[1] Weiss AJ, Barrett ML, Heslin KC, Stocks C. Trends in Emergency Department Visits Involving Mental and Substance Use Disorders, 2006-2013. HCUP Statistical Brief \#216. December 2016. Agency for Healthcare Research and Quality, Rockville, MD. http://www. hcup-us.ahrq.gov/reports/statbriefs/sb216-Mental-Substance-UseDisorder-ED-Visit-Trends.pdf.

[2] Liljeqvist Hennign et al. BMC Medical Informatics and Decision Making 2014, 14:84 http://www.biomedcentral.com/1472-6947/14/84.

[3] Gould DW, Walker D, and Yoon PW. The Evolution of BioSense: Lessons Learned and Future Directions. Public Health Reports 2017, Vol. 132(Supplement I)

\section{*Achintya N. Dey}

E-mail: aad2@cdc.gov 